\title{
Divergência genética entre codornas de corte para características de produção
}

\author{
Genetic divergence between meat type quails for performance traits
}

\author{
Rogério de Carvalho Veloso ${ }^{\mathrm{I}}$ Talita Andrade Ferreira ${ }^{\mathrm{II}^{*}}$ Eduardo Silva Cordeiro Drumond $^{\mathrm{II}}$ \\ Aldrin Vieira Pires ${ }^{I I}$ Jéssica Amaral MirandaII Leonardo Silva Costa ${ }^{\text {II }}$ \\ Luiza Rodrigues Alves Abreu ${ }^{\text {II }}$ Cristina Moreira BonaféII Sandra Regina Freitas Pinheiro ${ }^{\text {II }}$ \\ Idalmo Garcia Pereira ${ }^{\text {III }}$
}

RESUMO

Objetivou-se verificar a divergência genética entre linhagens de codornas de corte e seus cruzamentos para as características de desempenho através da análise multivariada. Foram utilizadas quatro linhagens de codornas de corte em um sistema de cruzamentos dialélicos completos, proporcionando a formação de 16 grupos de progênies, sendo quatro parentais puros: L1, L2, L3 e L4; seis grupos genéticos mestiços F1: G12, G13, G14, G23, G24 e G34; e seis grupos genéticos mestiços F1 reciprocos. Foram avaliadas as seguintes variáveis: peso corporal médio ao nascimento, aos 28, aos 35 e aos 42 dias de idade; o consumo médio de dieta do nascimento aos 35 e aos 42 dias de idade; a conversão alimentar do nascimento aos 35 e aos 42 dias de idade; e o ganho em peso do nascimento aos 35 e aos 42 dias de idade. $O$ desempenho dos grupos genéticos foi avaliado por meio da análise de variância multivariada e a primeira variável canônica, usando os testes do maior autovalor de Roy e da união-interseção de Roy para as comparações múltiplas. $O$ estudo da divergência genética foi feito por meio da análise por variáveis canônicas e pelo método de otimização de Tocher. As três primeiras variáveis canônicas explicaram $88,82 \%$ da variação entre os grupos genéticos. A divergência genética entre os grupos genéticos avaliados permitiu a formação de quatro grupos: 1 - L1, G12, G23, G21 e G41; 2 - G13 e G31; 3 - L4 e G43; 4 - G32; 5 G14 e G24; 6 - G42 e 7 - L2, L3 e G34, identificando a L4 como a linhagem que mais influiu na formação de novo grupos.

Palavras-chave: agrupamento, análise discriminante, variável canônica.

\section{ABSTRACT}

The objective was to evaluate the genetic divergence between lineages of quail and their crosses to the performance characteristics through multivariate analysis. Four lines of quails were used in a complete diallel system, provided training to 16 groups of progenies, four parental pure: L1, L2, L3 and L4; six genetic groups crossbred F1: G12, G13, G14, G23, G24 and G34; and six reciprocal F1 crossbred genetic groups. The variables were evaluated: mean body weight at birth, at 28 , at 35 and 42 days of age; the average consumption of diet from birth to 35 and 42 days of age; feed conversion birth at 35 and 42 days of age; and the weight gain from birth to 35 and 42 days of age. The performance of genetic groups was assessed by multivariate analysis of variance and the first canonical variable, using the tests of the largest eigenvalue of Roy and Roy union-intersection for multiple comparisons. The study of genetic diversity was done by canonical variate analysis and by the optimization method of Tocher. The first three canonical variables explained $88.82 \%$ of the genetic variation between groups. The genetic divergence between the analyzed genetic groups allowed the formation of four groups: 1 - L1, G12, G23, G21 and G41; 2 - G13 and G31; 3 - L4 and G43; 4 - G32; 5 - G14 and G24;6 - G42 and 7 L2, L3, identifing L4 G34 as the lineage that most influenced the formation of new groups.

Key words: canonical variable, discriminant analysis, group.

\section{INTRODUÇÃO}

O melhoramento genético tem contribuído de forma expressiva para a evolução da avicultura de corte no Brasil, sendo que a coturnicultura vem ganhando cada vez mais destaque e com números crescentes, levando assim a uma demanda por material genético de qualidade. Contudo, a primeira dificuldade encontrada pelo melhorista é o

\footnotetext{
IPrograma de Pós-graduação em Zootecnia (PPGZOO), Universidade Federal de Viçosa (UFV), Viçosa, MG, Brasil.

ID Departamento de Zootecnia (DZO), Universidade Federal dos Vales do Jequitinhonha e Mucuri (UFVJM), 39100-000, Diamantina, MG, Brasil. E-mail: tatilitazoo@gmail.com. *Autor para correspondência.

${ }^{\mathrm{III} D e p a r t a m e n t o ~ d e ~ Z o o t e c n i a ~(D Z O), ~ U n i v e r s i d a d e ~ F e d e r a l ~ d e ~ M i n a s ~ G e r a i s ~(U F M G), ~ B e l o ~ H o r i z o n t e, ~ M G, ~ B r a s i l . ~}$ Recebido 03.12.13 Aprovado 02.12.14 Devolvido pelo autor 14.03.15
} CR-2013-1590.R3 
conhecimento e identificação dos grupos genéticos que serão utilizados nos cruzamentos, como linhas machos e linhas fêmeas.

Nesse sentido, o estudo de divergência genética desempenha papel importante na avaliação de grupos genéticos, pois permite avaliar a superioridade de alguns genótipos sobre outros e identificar genótipos divergentes que possam ser utilizados como progenitores em programas de hibridação, evitando assim a consanguinidade e consequentemente a depressão de características determinantes da viabilidade da espécie, tais como taxa reprodutiva e sobrevivência (FALCONER, 1981). Ainda é importante ressaltar que o grupo genético tem efeito direto nas características de desempenho (CORREAA et al., 2007; VELOSO et al., 2012; TEIXEIRA et al., 2013) e podem interferir na rentabilidade do sistema.

Para predizer a divergência genética, várias técnicas de análise multivariada podem ser aplicadas, entre as quais, a análise de agrupamento, que tem por finalidade reunir, por algum critério de classificação, os progenitores em vários grupos, de modo que haja homogeneidade dentro do grupo e heterogeneidade entre grupos (CRUZ et al., 2004).

A utilização de técnicas multivariadas é mais apropriada em virtude das características serem consideradas simultaneamente, pois levam em consideração as possíveis correlações entre as características investigadas (VENTURA et al., 2012).

As técnicas de análise de agrupamento iniciam-se com o cálculo da matriz de similaridade ou dissimilaridade entre as unidades amostrais. A distância de Mahalanobis $\left(\mathrm{D}^{2}\right)$ é determinada usando as características fenotípicas e tem sido recomendada e utilizada por outros autores (YAMAKI et al., 2008; VENTURA et al., 2012). Uma vez que a $D^{2}$ foi determinada, o método de otimização de Tocher (RAO, 1952) tem sido uma alternativa para o agrupamento das unidades amostrais, que consiste em manter sempre uma distância intergrupo maior do que a distância média intragrupo (CRUZ et al., 2004).

Trabalhos na literatura, utilizando análise multivariada em codornas de corte, ainda são escassos. Assim, objetivou-se com este trabalho verificar a divergência genética entre quatro linhagens de codornas de corte, e seus cruzamentos, utilizando as características de desempenho por meio de técnicas de análise multivariada.

\section{MATERIAL E MÉTODOS}

Foram utilizadas quatro linhagens de codornas de corte (Coturnix coturnix), denominadas
L1, L2, L3 e L4, em um sistema de cruzamentos dialélicos completos, proporcionando a formação de 16 grupos de progênies, sendo quatro parentais puros (L1, L2, L3 e L4), seis grupos genéticos mestiços F1 (G12, G13, G14, G23, G24 e G34) e seis grupos genéticos mestiços $F 1$ recíprocos $(\mathrm{G} 21, \mathrm{G} 31, \mathrm{G} 41$, G32, G42 e G43). Esses 16 grupos de progênie foram distribuídos em um delineamento inteiramente casualizado, com três repetições, contendo 20 aves não sexadas por unidade experimental, totalizando 960 codornas. Informações sobre as linhagens utilizadas neste trabalho podem ser encontradas em DRUMOND et al. (2014); TEIXEIRA, et al. (2013) e VELOSO et al. (2012).

As codornas foram criadas em gaiolas coletivas do tipo bateria com dimensões de $60 \mathrm{x}$ $60 \times 33 \mathrm{~cm}$ de altura, sendo um total de três baterias com 16 gaiolas cada, recebendo água e dieta à vontade durante todo o período experimental, sendo que a dieta continha $25 \%$ PB e $2900 \mathrm{kcal}$ de EM $\mathrm{kg}^{-1}$, do nascimento ao $21^{\circ}$ dia de idade, e $24 \% \mathrm{~PB}$ e $2925 \mathrm{kcal}^{\mathrm{de}} \mathrm{EM} \mathrm{kg}^{-1}$, do $22^{\circ}$ ao $42^{\circ}$ dia de idade, segundo recomendações de exigências nutricionais da literatura nacional (CORRÊA et al., 2007; CORRÊA et al., 2008; VELOSO et al., 2012).

Como fonte de aquecimento, foram utilizadas lâmpadas de 100watts em todas as gaiolas até a segunda semana de idade. As temperaturas mínima e máxima médias, registradas durante o período experimental, foram de 24,1 e $29,5^{\circ} \mathrm{C}$, respectivamente.

Foram avaliados o peso corporal médio ao nascimento (PN), aos 28 (P28), aos 35 (P35) e aos 42 dias de idade (P42); o consumo médio de dieta do nascimento aos 35 dias de idade (CMD35) e aos 42 dias de idade (CMD42); a conversão alimentar do nascimento aos 35 (CA35) e aos 42 dias de idade (CA42); e o ganho em peso do nascimento aos 35 (GP35) e aos 42 dias de idade (GP42).

As análises estatísticas foram feitas utilizando-se o procedimento "PROC CANDISC" do programa SAS (1990), segundo o modelo: $y_{i j k}$ $=u_{k}+\mathrm{G}_{i k}+e_{i j k}$, em que: $y_{i j k}=$ valor observado da característica $\mathrm{k}$, na repetição $\mathrm{j}$, do grupo genético i $(\mathrm{i}=1,2, \ldots, 16 ; \mathrm{j}=1,2,3 ; \mathrm{k}=1,2, \ldots, 10) ; u_{k}=$ média da característica $\mathrm{k} ; \mathrm{G}_{i k}=$ efeito do grupo genético i na característica $\mathrm{k} ; e_{i j k}=$ efeito do erro aleatório associado a cada observação: $y_{i j k}$.

Admite-se que os erros têm distribuição multinormal p-dimensional com vetor nulo de médias e matriz de variâncias e covariâncias $a$, comum a todas as combinações $i$ e $j$. Os $e_{i j k}$ correspondentes a diferentes unidades experimentais são independentemente distribuídos. 
Foi adotado o critério de descarte das variáveis de acordo com a avaliação da importância das características, a partir dos coeficientes de ponderação de cada característica nas últimas variáveis canônicas que, por estimação, retém proporção mínima da variação total (CRUZ \& REGAZZI, 1997). Após o descarte dessas variáveis, foram feitos os testes de médias para a comparação entre os grupos genéticos com as características que foram mantidas.

A hipótese testada pela MANOVA é a de igualdade dos vetores de médias entre os grupos genéticos, ou seja: $\mathrm{H}_{\mathrm{O}}=\mathrm{L}_{1}=\mathrm{G}_{12}=\mathrm{G}_{13}=\mathrm{G}_{14}=\mathrm{L}_{2}=\mathrm{G}_{21}=\cdots=\mathrm{L}_{4}$.

Para testar essa hipótese, foi utilizado o teste do maior autovalor de Roy, que consiste na determinação das raízes características (autovalores) da equação característica $\left|E^{-1} \lambda I\right|=0$ ou $|H-\lambda E|=0$ em que: $1=$ matriz identidade; $\lambda$ autovalores; $\mathrm{E}^{-1}=$ inversa da matriz de soma de quadrados e produtos residuais; e $\mathrm{H}=$ matriz de soma de quadrados e produtos de tratamentos. Sendo $\lambda_{\max }$ o maior autovalor de $\mathrm{E}^{-1} \mathrm{H}$, define-se a estatística $\theta \frac{\max }{1 \text { max }}$.

O valor assim obtido $(\theta)$ é comparado com o limite de significância fornecido em tabelas (MORRISON, 1967; HARRIS, 1975).

A seguir, realizou-se o teste de comparações de contrastes entre médias, pelo teste da união-interseção de Roy. A análise de agrupamento foi conduzida adotando-se a $\mathrm{D}^{2}$ (utilizando-se o procedimento "PROC CANDISC" do programa SAS (1990) como medida de dissimilaridade, sobre a qual foi empregado o método de agrupamento de otimização de Tocher, conforme citado por RAO (1952). Para o agrupamento, trabalhou-se com a matriz de distâncias entre pares de grupos genéticos, que é simétrica, com zeros na diagonal principal. Inicialmente, foi identificado o par de grupos genéticos com menor $\mathrm{D}^{2}$, na matriz das distâncias. Esses dois grupos genéticos deram então origem ao primeiro grupo. A partir daí, foi avaliada a possibilidade de inclusão de mais um genótipo (o mais próximo ao grupo recém-formado), obedecendo ao critério anterior (PIASSI et al., 1995).

Esquematicamente, mostra-se o critério para formação do agrupamento: $D_{(i j) k}^{2}=D_{i k}^{2}+D_{j k}^{2}$ ou $D_{(i j k) l}^{2}=D_{(i j) l}^{2}+D_{k l}^{2} . \operatorname{Se}_{\frac{D^{2}(G r u p o)}{g}} \leq \theta$ inclui-se o grupo genético no grupo; se $\frac{D^{2}(\text { Grupo })}{g}>\theta$ não inclui grupo genético no grupo; em que: $\theta=$ limite de acréscimo adotado, $\mathrm{j}$, $\mathrm{k}$ e l= grupos genéticos do grupo; $\mathrm{i}=$ grupo genético a ser incluído, ou não, no grupo; g= número de grupos genéticos que formam o grupo que está sendo formado, conforme YAMAKI et al. (2008).
Foi estimada a função discriminante, baseada na variável canônica de maior correlação com os dados, primeira variável canônica, obtendo um escore para cada genótipo. A partir desses escores, representando os genótipos de forma unidimensional, realizou-se uma análise de variância (ANOVA) para testar a hipótese de igualdade dos genótipos. Para testar contrastes entre as médias, foram calculadas as diferenças mínimas significativas (DMS) ao nível de probabilidade de $5 \%$ pelo teste de Roy (MORRISON, 1967; HARRIS, 1975).

\section{RESULTADOS E DISCUSSÃO}

O princípio fundamental do descarte de variáveis demonstra que a variância das variáveis canônicas decresce da primeira para a última. Tem-se então que as últimas variáveis canônicas são responsáveis pela explicação de uma fração muito pequena da variância total. Sendo assim, as características P42 e GP42 foram passíveis de descarte, pois apresentam os maiores valores absolutos nas últimas variáveis canônicas (can9 = 2255,82 e can $8=821,04$ ) e representam, portanto, uma variação praticamente insignificante (CRUZ \& REGAZZI, 1997). Ainda pode ser observado que, a partir da variável canônica 8 (can8), não foi descartada mais nenhuma característica, pois os maiores valores absolutos das demais variáveis canônicas se repetiram para a GP42, que já havia sido descartada anteriormente, demonstrando que seria redundante tomar essa medida em futuros trabalhos de divergência genética nas condições ambientais testadas. Isso ocorre porque, nesse período, as aves já se encontram no estágio final de produção, direcionadas para o abate, tornando essas informações redundantes. Acrescenta-se ainda o fato de atualmente haver forte tendência de prática de abate de codornas de corte aos 35 dias de idade, pois até esta idade a taxa relativa de síntese proteica é maior no animal, enquanto a taxa relativa de deposição de gordura tende a ser maior com o aumento da idade do animal (GONZALEZ \& SARTORI, 2002).

Considerando apenas as características não descartadas, ou seja, aquelas que mais explicam a variância total, não foram observadas diferenças significativas $(\mathrm{P}>0,05)$ entre os genótipos pelo teste de Roy, para todas as características (Tabela 1), pois esse teste supera o poder do teste de razão de verossimilhança, quando o maior autovalor exceder muito os outros e for equivalente para amostras de tamanho grande, além de descrever prontamente a fonte de qualquer diferença estatisticamente significativa, por meio das funções 
Tabela 1 - Média das características não descartadas para os grupos genéticos de codornas de corte.

\begin{tabular}{|c|c|c|c|c|}
\hline \multirow{2}{*}{ Grupo genético } & \multicolumn{4}{|c|}{ 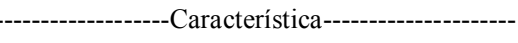 } \\
\hline & $\mathrm{PN}(\mathrm{g})$ & P28 (g) & P35 (g) & GP35 (g) \\
\hline L1 & 9,96 & 192,34 & 235,36 & 225,40 \\
\hline G12 & 9,97 & 201,93 & 247,31 & 237,34 \\
\hline G13 & 9,40 & 171,83 & 212,84 & 203,44 \\
\hline G14 & 9,74 & 192,83 & 226,92 & 217,17 \\
\hline G21 & 9,84 & 186,63 & 232,57 & 222,73 \\
\hline L2 & 8,46 & 184,20 & 232,75 & 224,30 \\
\hline G23 & 9,88 & 180,50 & 221,87 & 211,98 \\
\hline G24 & 9,11 & 191,74 & 235,51 & 226,40 \\
\hline G31 & 9,38 & 182,47 & 226,45 & 217,07 \\
\hline G32 & 9,05 & 170,00 & 188,30 & 179,25 \\
\hline L3 & 8,96 & 164,53 & 203,33 & 194,37 \\
\hline G34 & 8,58 & 175,37 & 216,64 & 208,05 \\
\hline G41 & 10,18 & 202,19 & 246,64 & 236,47 \\
\hline G42 & 8,56 & 201,65 & 249,89 & 241,33 \\
\hline G43 & 9,76 & 170,13 & 208,17 & 198,41 \\
\hline L4 & 9,96 & 174,57 & 201,56 & 191,60 \\
\hline \multirow{2}{*}{ Grupo genético } & & -Caracte & ística------ & --------- \\
\hline & CMD35 & CA35 & CMD42 & CA42 \\
\hline L1 & 586,32 & 2,65 & 782,02 & 3,11 \\
\hline G12 & 696,94 & 3,00 & 939,87 & 3,42 \\
\hline G13 & 543,12 & 2,67 & 728,31 & 3,13 \\
\hline G14 & 617,05 & 2,88 & 804,53 & 3,33 \\
\hline G21 & 594,19 & 2,68 & 828,51 & 3,26 \\
\hline L2 & 586,42 & 2,64 & 790,44 & 3,07 \\
\hline G23 & 586,74 & 2,79 & 785,61 & 3,23 \\
\hline G24 & 585,38 & 2,59 & 797,82 & 3,06 \\
\hline G31 & 539,50 & 2,49 & 789,05 & 3,10 \\
\hline G32 & 452,17 & 2,56 & 676,06 & 3,14 \\
\hline L3 & 640,74 & 3,36 & 820,42 & 3,84 \\
\hline G34 & 612,93 & 2,95 & 844,46 & 3,49 \\
\hline G41 & 634,26 & 2,70 & 858,56 & 3,21 \\
\hline G42 & 653,71 & 2,74 & 890,33 & 3,19 \\
\hline G43 & 558,80 & 2,84 & 805,80 & 3,30 \\
\hline L4 & 535,35 & 2,78 & 760,81 & 3,40 \\
\hline
\end{tabular}

PN = Peso médio ao nascimento; P28 = Peso aos 28 dias de idade; P35 = Peso aos 35 dias de idade; CMD35 = Consumo médio de dieta aos 35 dias de idade; CA35 = Conversão alimentar aos 35 dias de idade; CMD42 = Consumo médio de dieta aos 42 dias de idade; CA42 = Conversão alimentar aos 42 dias de idade.

discriminantes estatisticamente significativas. Portanto, ele é mais exigente na rejeição das hipóteses de nulidade dos fatores (HARRIS, 1975). As médias para o PN, P28, P35, CMD35, CA35, CMD42 e CA42 (Tabela 1) são semelhantes às de diversos trabalhos relatados na literatura (CORRÊA et al., 2007; CORRÊA et al., 2008 e OTUTUMI et al., 2009).

A homogeneidade de desempenho entre os genótipos avaliados foi confirmada após análise de variância e utilização do teste de Roy, aplicados à função discriminante baseada na variável canônica de maior correlação com os dados para testar a hipótese de igualdade dos genótipos. Nesse sentido, verificouse que não houve diferença $(\mathrm{P}>0,05)$ no desempenho entre os grupos genéticos avaliados (Tabela 2). Pelo escore da primeira variável canônica, conseguese ter uma visão aproximada do comportamento geral do grupo genético, considerando-se todas as características agrupadas em um único índice (PIRES et al., 2002).

A verificação da divergência entre os grupos genéticos em diagramas de dispersão é possível quando as variáveis canônicas explicam pelo menos $80 \%$ da variância total. As três primeiras variáveis canônicas (VC) explicaram $88,82 \%$ da variação observada $\left(\mathrm{VC}_{1}-52,78 \% ; \quad \mathrm{VC}_{2}-27,07 \%\right.$; $\mathrm{VC}_{3}-8,97 \%$ ), sendo considerada eficiente, pois a maior parte da variação entre as características pode ser explicada nas três primeiras variáveis canônicas (CRUZ \& REGAZZI, 1997).

A dispersão gráfica foi realizada utilizando as duas primeiras médias canônicas para que haja uma melhor compreensão da distribuição dos grupos genéticos (Figura 1). Através do método de otimização de Tocher, verificou-se a formação de sete grupamentos genéticos, permitindo evidenciar a similaridade genética dentro dos grupos e a divergência genética entre estes. O grupo 1 foi constituído pelos grupos genéticos: L1, G12, G23, G21 e G41; grupo 2: G13 e G31; grupo 3: L4, e G43; grupo 4: G32; grupo 5: G14 e G24; grupo 6: G42 e grupo 7: L2, L3 e G34. O método de otimização de Tocher tem sido bastante utilizado e pormenorizado em PIRES et al., 2002; YAMAKI et al., 2008; VENTURA et al., 2012. Em um programa de melhoramento, é desejável que se dê prioridade ao cruzamento de materiais que tenham elevada média e apresentam diversidade genética entre si, ou seja, cruzar bons e divergentes, para que identifiquem materiais bons que se complementem e dessa forma façam usar da fração não aditiva existente na variância genética.

É interessante notar que, para a formação dos grupos, houve propensão sobre alguns grupos genéticos, alterando de acordo com os machos ou fêmeas das linhagens utilizadas. $\mathrm{Na}$ formação do grupo 1, os machos da L1 e da L2 e as fêmeas da L1 foram predominantes no sistema de cruzamento, o que nos leva a predizer que os machos da L2 estão mais próximos geneticamente da L1. No grupo 2, não há diferença se a linhagem macho ou fêmea for a L1 ou a L3 para formação dos cruzamentos, mostrando assim a similaridade genética entre os grupos genéticos mestiços 
Tabela 2 - Escores e diferença mínima significativa (DMS) para as linhagens de codorna de corte, com base na função discriminante de Fischer (FDF), pelo teste de Roy.

\begin{tabular}{lc}
\hline Linhagem & Escores da FDF \\
\hline L11 & $-0,0255$ \\
L12 & $-0,0274$ \\
L13 & $-0,0294$ \\
L14 & $-0,0317$ \\
L21 & $-0,0249$ \\
L22 & $-0,0483$ \\
L23 & $-0,0256$ \\
L24 & $-0,0366$ \\
L31 & $-0,0296$ \\
L32 & $-0,0335$ \\
L33 & $-0,0501$ \\
L34 & $-0,0474$ \\
L41 & $-0,0212$ \\
L42 & $-0,0515$ \\
L43 & $-0,0245$ \\
L44 & $-0,0199$ \\
DMS & 0,0929 \\
\hline
\end{tabular}

F1 e os grupos genéticos mestiços F1 recíprocos (G13 e G31, respectivamente). Ao observar o grupo 3, notase a preferência pelos machos da L4 nos sistemas de cruzamento, assim, pode-se inferir que a L4 e o seu grupo genético mestiço $\mathrm{F} 1$ recíproco $(\mathrm{G} 43)$ estão próximos geneticamente. Nos grupos 4 e 6 , a diferenciação dos grupos se deu pela linha macho utilizada (L3 e L4 respectivamente), mostrando assim a dissimilaridade ou heterogeneidade genética entre os machos da L3 e da
L4 para a formação dos respectivos grupos. Entretanto, a formação do grupo 5 se deu principalmente pela utilização das fêmeas L4, mostrando assim que as fềmeas da L4 foram determinantes para a formação do grupo. O grupo 7 foi constituído principalmente pelas linhas puras L2 e L3, mais o G34, evidenciando assim a similaridade genética da L2 com a L3. Esse tipo de informação permitiu identificar que a L4 foi a linhagem que mais influenciou a formação de novos grupos e que apresentou a melhor capacidade combinatória em nível expressivo para produzirem, em alta frequência, recombinações favoráveis para seleção de genitores, sendo extremamente útil no estabelecimento dos cruzamentos a serem realizados.

\section{CONCLUSÃO}

A análise multivariada mostrou ser uma ferramenta adequada para indicar as características que são passíveis de descarte: peso corporal aos 42 dias de idade e ganho em peso do nascimento aos 42 dias de idade.

A hipótese de homogeneidade entre os cruzamentos realizados foi confirmada, porém, por meio da distância Mahalanobis, foi possível formar sete grupos distintos, evidenciando diversidade genética entre estes e identificando a L4 como a linhagem que mais influiu na formação de novo grupos e apresentou a melhor capacidade combinatória em nível expressivo para produzirem, em alta frequência, recombinações favoráveis para seleção de genitores.

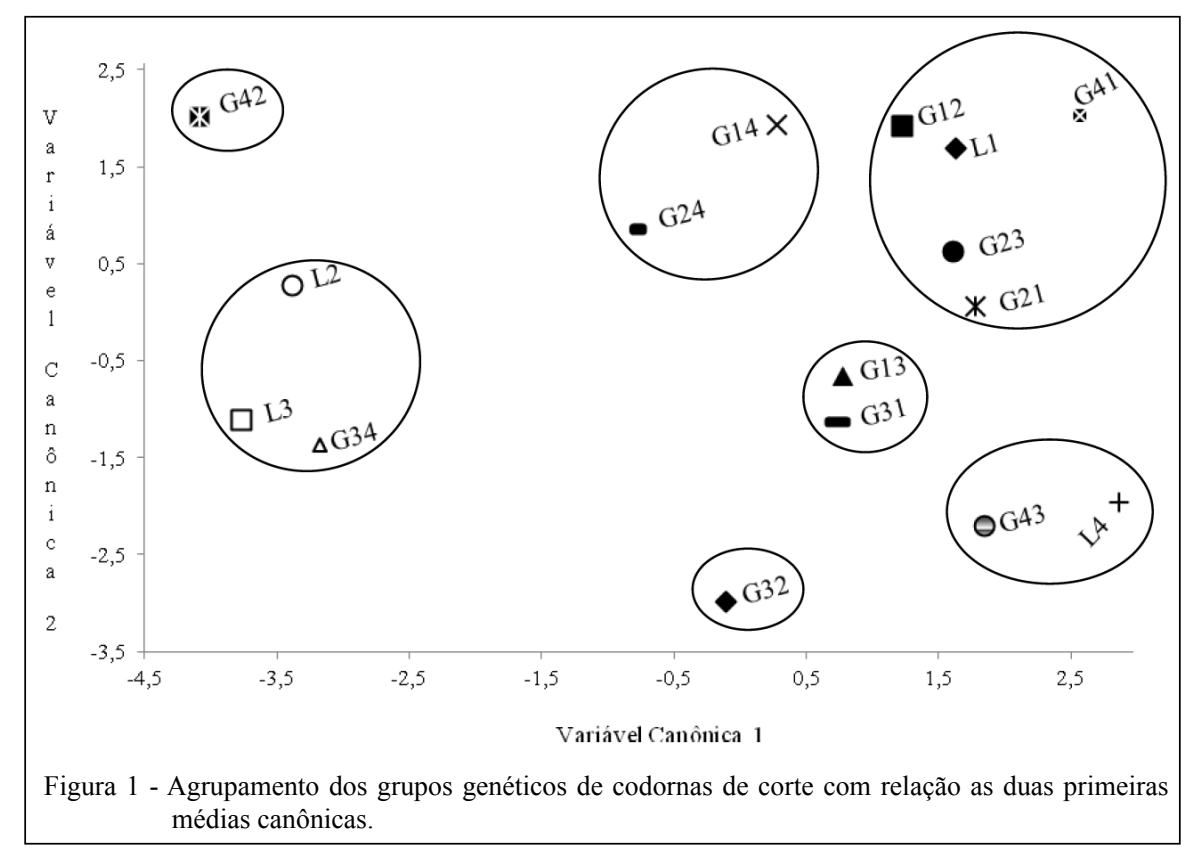

Ciência Rural, v.45, n.8, ago, 2015. 


\section{AGRADECIMENTOS}

Os autores agradecem ao apoio recebido da Coordenação de Aperfeiçoamento de Pessoal de Nível Superior (CAPES), Conselho Nacional de Desenvolvimento Científico e Tecnológico (CNPq) e Fundação de Amparo à Pesquisa do Estado de Minas Gerais (FAPEMIG)

\section{COMITÊ DE ÉTICA E BIOSSEGURANÇA}

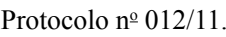

\section{REFERÊNCIAS}

CORRÊA, G.S.S. et al. Exigência de proteína bruta e energia metabolizável para codornas de corte EV1. Arquivo Brasileiro de Medicina Veterinária e Zootecnia, v.59, n.3, p.797-804, 2007. Disponível em: <http://dx.doi.org/10.1590/S010209352007000300035>. Acesso em: 12 ago. 2013. doi: 10.1590/ S0102-09352007000300035.

CORRÊA, G.S.S. et al. Níveis de proteína bruta para codornas de corte durante o período de crescimento. Arquivo Brasileiro de Medicina Veterinária e Zootecnia, v.60, n.1, p.209-217, 2008. Disponível em: <http://dx.doi.org/10.1590/S010209352008000100029>. Acesso em: 10 set. 2013. doi: 10.1590/ S0102-09352008000100029.

CRUZ, C.D. et al. Modelos biométricos aplicados ao melhoramento genético. Viçosa, MG: UFV, 1997. 475p.

CRUZ, C.D. et al. Modelos biométricos aplicados ao melhoramento genético. 3.ed. Viçosa: UFV, 2004. v.1, 480p.

DRUMOND, E.S.C. et al. Rendimento de carcaça de codornas de corte em cruzamentos dialélicos. Ciência Rural, v.44, n.1, p.129134, 2014. Disponível em: <http://dx.doi.org/10.1590/S010384782013005000143>. Acesso em: 21 mar. 2014. doi: 10.1590/ S0103-84782013005000143.

FALCONER, D.S. Introdução à genética quantitativa. Tradução de SILVA, M.A.; SILVA, J.C. Viçosa, MG, UFV, 1981. 279p.

GONZALEZ, E.; SARTORI, J.R. Crescimento e metabolismo muscular. In: MACARI, M.; FURLAN, R.L.; GONZALES, E. (Eds.) Fisiologia aviária aplicada a frangos de corte. Jaboticabal: FUNEP/UNESP, 2002. 375p.

HARRIS, R.J. A primer of multivariate statistics. New York: Academic, 1975. 332p.
MORRISON, D.F. Multivariate statistical methods. New York: McGraw- Hill, 1967. 338p.

OTUTUMI, L.K. et al. Efeito do probiótico sobre o desempenho, rendimento de carcaça e exigências nutricionais de proteína bruta de codornas de corte. Revista Brasileira de Zootecnia, v.38, n.2, p.299-306, 2009. Disponível em: <http://dx.doi.org/10.1590/ S1516-35982009000200012>. Acesso em: 15 set. 2013. doi: 10.1590/S1516-35982009000200012.

PIASSI, M. et al. Estudo da divergência entre oito grupos de aves de postura, por meio de técnicas de análise multivariada. Revista Brasileira de Zootecnia, v.24, n.5, p.715-727, 1995. Disponível em: <http://www.sbz.org.br/revista/artigos/382.pdf >. Acesso em: 24 mar. 2014.

PIRES, A.V. et al. Estudo da divergência genética entre as raças suínas Duroc, Landrace e Large White, utilizando técnicas de análise multivariada. Archivos Latinoamericano de Produção Animal, v.10, n.2, p.81-85, 2002. Disponível em: <http://www. alpa.org.ve/PDF/Arch\%2010-2/arch-2.pdf>. Acesso em: 08 ago. 2013.

RAO, C.R. Advanced statistical methods in biometric research. New York: Jonh Wiley \& Sons, 1952. 390p.

SAS Institute. SAS/STAT ${ }^{\circledR}$ user's guide: Version 9.1, Cary: 2002. v.2, 1052p.

TEIXEIRA, B.B. et al. Desempenho de codornas de corte submetidas a diferentes níveis de proteína bruta e energia metabolizável. Ciência Rural, v.43, n.3, p.524-529, 2013. Disponível em: <http:// dx.doi.org/10.1590/S0103-84782013005000014>. Acesso em: 10 mar. 2014. doi: 10.1590/S0103-84782013005000014.

VELOSO, R.C. et al. Níveis de proteína bruta e energia metabolizável em uma linhagem de codorna de corte. Acta Scientiarum Animal Sciences, v.34, n.2, p.169-174, 2012. Disponível em: <http://dx.doi.org/10.4025/actascianimsci. v34i2.12589>. Acesso em: 10 ago. 2013. doi: 10.4025/ actascianimsci.v34i2.12589

VENTURA, H.T. et al. Use of multivariate analysis to evaluate genetic groups of pigs for dry-cured ham production. Livestock Science, v.148, p.214-220, 2012. Disponível em: <http://dx.doi. org/10.1016/j.livsci.2012.06.007>. Acesso em: 15 ago. 2013. doi: 10.1016/j.livsci.2012.06.007.

YAMAKI, M. et al. Divergência genética entre linhagens de matrizes de corte por meio de análise de agrupamento. Revista Brasileira de Zootecnia, v.37, n.5, p.829-833. 2008. Disponível em: <http:// dx.doi.org/10.1590/S1516-35982008000500008>. Acesso em: 12 set. 2013 doi: 10.1590/S1516-35982008000500008. 